\title{
FENOLOGIA E PRODUTIVIDADE DO ARAÇÁ-BOI (Eugenia stipitata, MYRTACEAE) NA AMAZÔNIA CENTRAL
}

\author{
Martha de Aguiar FALCÃO', Rozana de M. Souza GALVÃO², Charles R. CLEM-
} ENT $^{3}$, Sidney A. do N. FERREIRA ${ }^{3}$, Sebastiana das Graças SAMPAIO ${ }^{2}$

RESUMO - O araçá-boi (Eugenia stipitata McVaugh ssp, sororia McVaugh, Myrtaceae) é uma frutífera nativa da Amazônia Ocidental com potencial para a indústria de sucos e flavorizantes. Embora pouco plantada na Amazônia brasileira devido a sua acidez, é frequentemente cultivada na Amazônia peruana. O conhecimento de sua fenologia pode ajudar no planejamento do manejo do plantio e da comercialização dos frutos. A fenologia de dez plantas, crescendo num latossolo amarelo degradado, foi observada durante cinco anos. $\mathrm{O}$ araçá-boi geralmente floresceu e frutificou três vezes ao longo do ano e sempre teve pelo menos um pico de floração forte durante a estação seca (julho a setembro) e um pico de frutificaçào mais acentuado na estaçào chuvosa (janeiro a março). A floração é um evento complexo e demorado que pode durar de dois a três meses, embora o período entre o aparecimento do botão floral até a antese do flor é curto ( $\sim 15$ dias $)$ c o período entre a antese e a maturação dos frutos dura 50 a 60 dias. As regressōes múltiplas usadas para determinar o efeito das variáveis climáticas na floração e frutificação não apresentaram altos coeficientes de determinação, embora os modelos tenham sido significantes, provavelmente porque $o$ araçá-boi floresce várias vezes durante o ano e ainda não se sabe qual o estímulo mais importante para iniciar o processo. O vingamento dos frutos variou de menos de $5 \%$ a aproximadamente 15\%. O peso médio dos frutos avaliados em janeiro de 1988 foi $135 \mathrm{~g}$, com $77 \%$ de polpa. Ao longo do periodo, estimou-se que as dez plantas produziram em média 1000 frutos/ano, com uma mediana de 890 frutos/ano. Os insetos visitantes eram principalmente abelhas, especialmente Apis mellifera, Eulaema mocsaryi e Ptilotrigona lurida.

Palavras-chave: floração, frutificação, mudança foliar, vingamento, clima.

\section{Phenology and Yield of Araçá-boi (Eugenia stipitata, Myrtaceae) in Central Amazonia}

ABSTRACT - The araçá-boi (Eugenia stipitata McVaugh ssp. sororia McVaugh, Myrtaceae) is a small fruit species native to western Amazonia with international potential as a juice and flavor. Although seldom planted in Brazilian Amazonia because of its acidity, the species is being cultivated in other countries. A knowledge of phenology can help plan plantation management and fruit commercialization. The phenology of ten plants growing in a degraded oxisol was observed during five years. The araçá-boi generally flowered and fruited three times during the year, with at least one strong flowering peak in the dry season (July-September) and a strong fruiting peak in the rainy season (January-March). Flower initiation is a complex event that appears to take two to three months, although the period from flower bud appearance to anthesis is short ( $\sim 15$ days) and the period from anthesis to fruit maturation takes between 50 and 60 days. The multiple regressions used to determine the effect of climatic variables on flowering and fruiting had low determination coefficients, although the models were significant, probably because the araça-boi flowers several times during the year and the most important stimulous for flowering is still unknown. Fruit set varied from less than $5 \%$ to about $15 \%$, Mean fruit weight in January 1988 was $135 \mathrm{~g}$, with $77 \%$ of pulp. During the 5 years, the 10 plants yielded 1000 fruits/year, with a median number of 890 fruits/year. Most of the insect visitors were bees, especially Apis mellifera, Eulaema mocsaryi and Ptilotrigona lurida.

Key-words: flowering, fruiting, leaf change, fruit set, climate.

'Bolsista do PCI do INPA, modalidade DTI, com apoio do CNPq. Cond. RRio Tupana, BI.A. Apto. 602. Av. Cel. Teixeira, 386 - Nova Esperança - Manaus, AM. Brasil 69030-481

${ }^{2}$ Dept" de Biologia, Instituto de Ciências Biológicas, Universidade do Amazonas, Av. Gen. Rodrigo

Otavio Jordào Ramos, 3000 - Coroado III, 69077-000 Manaus, AM. Brasil.

${ }^{3}$ Instituto Nacional de Pesquisas da Amazônia, Cx. Postal 478, 69011-970 Manaus, AM. Brasil.

ACTA AMAZONICA, 30(1): 9-21. 2000. 


\section{INTRODUÇÃO}

$\mathrm{O}$ araçá-boi (Eugenia stipitata McVaugh subsp. sororia McVaugh, Myrtaceae) é uma espécie frutífera semi-domesticada pelos amerindios da Amazônia Ocidental, embora pouco conhecida na Amazônia Brasileira. É provavelmente originário da Amazônia Peruana e da região ocidental da Amazônia Brasileira (Pinedo et al., 1981; Clement, 1989). Seu cultivo é economicamente promissor, pois o seu sabor é atrativo para o mercado internacional e tem grande potencial para processamento, inclusive misturado com outras frutas (Gentil \& Clement, 1996). Embora pouco plantada na Amazônia brasileira, devido principalmente à sua elevada acidez que não agrada o palador do povo, a espécie vem sendo cultivada em outros paises.

O araçá-boi é um arbusto bastante ramificado e densamente folhoso, com folhas elipticas e verdeescuras. As pequenas inflorescências possuem de três a dez flores hermafroditas, com pétalas brancas e 75 a 100 estames (McVaugh, 1956, 1958). O fruto é uma baga que varia de 30 a $800 \mathrm{~g}$, com peso médio de 200 $\mathrm{g}$, que exala um aroma muito agradável quando maduro. $\mathrm{O}$ pericarpo do fruto é amarelo-canário, aveludado e fino. A polpa é suculenta, de sabor agradável porém muito ácida. É utilizada na preparação de refrescos, sucos, compotas, cremes, geleías, e doces, raramente sendo consumida in natura devido à sua acidez. As sementes são de tamanho variável, com 3 a 20 por fruto (Falcão et al., 1988; Chávez \& Clement, 1984; Calzada, 1980; Cavalcante, 1991).

Wielgolaski (1974) definiu a fenologia como o estudo do efeito da periodicidade das condições climáticas, influenciado pelas condições edáficas e ecológicas em geral, sobre o cíclo biológico das plantas, especialmente sobre os orgãos reprodutivos e de crescimento vegetativo. O conhecimento da fenologia pode ajudar o planejamento do manejo do plantio e da comercialização dos frutos. Falcão $e t$ al. (1988) observaram que a floração em plantas juvenis de araçá-boi ocorreu durante o ano inteiro e a frutificação aconteceu periodicamente em seguida à floração. A emissão de botões florais aumentou sensivelmente com o início da estação seca, como também foi observado por Calzada (1980) e Pinedo et al. (1981).

O objetivo deste estudo foi avaliar a fenologia da Eugenia stipitata durante o periodo de 1985 a 1990, numa plantação que estava chegando a maturação fisiológica numa área aberta em ambiente urbano. Este período serve como complemento ao estudo anterior (Falcão et al., 1988), que teve duração de apenas dois anos e foi feito no puro início da fase reprodutiva da araçá-boi.

\section{MATERIAL E MÉTODOS}

O estudo foi iniciado em 1985 , em um plantio de araçá-boi com treze meses no campo, situado no Campus do V-8 do Instituto Nacional de Pesquisas da Amazônia (INPA), 
Manaus, Amazonas, Brasil. O germoplasma deste plantio foi oriundo do primeiro plantio experimental no INPA (Chávez \& Clement, 1984), que, por sua vez, foi oriundo do Instituto Nacional de Investigaciones Agropecuárias (INIA) em Iquitos, Perú.

O clima de Manaus é classificado com "Afi" no sistema de Köppen (Ribeiro 1976), com médias anuais de $2.419 \mathrm{~mm}$ de chuva, $26,7^{\circ} \mathrm{C}$ e $87,5 \%$ de umidade relativa do ar. Os dados climáticos de 1985 a 1990 foram obtidos do Ministério de Agricultura, $1^{\text {o }}$ Distrito de Meteorologia de Manaus, a $4 \mathrm{~km} \mathrm{da}$ área experimental (Fig. 1A). O solo da área experimental está classificado como Latossolo-Amarelo, textura argilosa, com relêvo suave ondulado (Newton Falcão, INPA, com. pess.). A área do plantio se encontrava em extrema degradação, pois antes do plantio vinha sendo usada como pasto por 30 anos, seguida por 10 anos de abandono (durante estes 10 anos a capoeira cresceu muito pouco, indicando severa degradação), seguida de limpeza da área por trator de esteira. Em 1984, o plantio do araçá-boi foi feito em covas adubadas com esterco e 100 $\mathrm{g}$ de superfosfato triplo. Durante o periodo das observações, o manejo do plantio consistiu apenas de roçagem e coroamento (capina) na área de projeção da copa, com a montoa do material roçado e capinado ao redor das plantas.

As observações fenológicas foram efetuadas semanalmente no periodo de abril de 1985 a dezembro de 1990, em 10 plantas escolhidas ao acaso dentre as 42 existentes no plantio. Foram escolhidos ao acaso três galhos de cada planta, onde contaram-se o número de flores e frutos (Falcão et al., 1988). Avaliouse ainda a mudança foliar de acordo com a metodologia de Falcão (1983). O total de flores e frutos por planta foi estimado pela regra de três simples com base no número de galhos na planta. A relação entre a floração, a frutificação e o clima foi estudada com regressão múltipla (Sokal \& Rohlf, 1969), variando o número de meses entre a variável climática e sua respectiva floração para determinar os prováveis estímulos da floração. A taxa de vingamento anual (número de frutos resultantes do número de flores observadas durante $o$ ano) foi estimada e comparada com a precipitação anual.

Em 1988, escolheram-se ao acaso dez botões florais de cada árvore, os quais foram revestidos com saquinhos de morim e observados durante 15 dias para determinar a taxa de auto-fecundação não assistida. Durante a primeira safra deste ano (janeiro) coletou-se uma amostra de frutos maduros de cada planta, os quais foram pesados inteiros, e depois suas sementes e cascas. As sementes de cada fruto foram contadas. A produtividade foi estimada considerando-se uma densidade de 400 plantas/ha, multiplicada pela mediana do número de frutos/planta, vezes o peso médio do fruto. A mediana foi usada em lugar da média porque a distribuição do número de frutos/planta foi anormal e porque o 
peso dos fruto foi avaliado uma única vez.

Amostras dos insetos visitantes das flores foram capturados entre cinco e dez horas da manhã em 1988, fixados e montados para identificação. O pólen retirado das patas dos insetos foi preparado de acordo com Erdtman (1960) e montado em lâmina-laminula para identificação.

\section{RESULTADOS E DISCUSSÃO}

\section{Floração}

$\mathrm{O}$ araçá-boi apresentou algumas flores durante a maior parte do ano, geralmente com dois ou três picos de maior intensidade de floração (Fig. 1B), embora em 1986 esses picos tenham sido pequenos. Um desses picos ocorreu sempre no período de menor precipitação (estação seca junho a setembro), enquanto os demais ocorreram logo após a estação seca (outubro a novembro 1985, 1986, outubro a dezembro 1987, novembro a dezembro 1989) ou no pleno estação chuvosa (dezembro 1985 a março 1986, janeiro a março 1987, novembro 1987 a fevereiro 1988, dezembro 1989 a fevereiro 1990). Observou-se que houve muita variação entre as dez plantas e que a variação era sempre maior durante os picos de floração (Fig. 1B).

Falcão et al. (1988) observaram plantas juvenis na Amazônia Central e Pinedo et al. (1981) plantas adultas na Amazônia Ocidental, e concluíram que $o$ araçá-boi floresce $o$ ano inteiro, com pequenos intervalos sem floração, embora a variação de planta a planta tenha sido quase sempre alto.
Os picos de floração observados por Falcão et al. (1988) chegaram a 800 flores/planta no segundo ano de reprodução, enquanto os observados aqui variaram de 1000 (1986) a 10.000 (1988); vale ressaltar aqui que estas estimativas podem ser altas ou baixas devido ao cálculo feito por regra de três. Assim como no presente trabalho, eles observaram ainda que os maiores picos de floração ocorreram durante os meses de menor precipitaçâo, sugerindo que esta é uma tendência em todas as áreas onde o araçá-boi é plantado (tanto na Amazônia Central como Ocidental; tanto em solos degradados como não degradados) e em plantas de qualquer idade reprodutiva.

Os botões florais são de rápido desenvolvimento; o intervalo entre seu aparecimento e a antese das flores é de aproximadamente 15 dias. As flores abrem pela manhã, duram pelo menos 24 horas, quando começam a murchar e, se não forem fecundadas, caem ao longo dos próximos dias. Se as flores forem fecundadas, as pétalas caem a partir do terceiro dia. Embora estas observações tenham sido feitas em um ecossistema quase urbano, são similares as de Falcão et al. (1988) feitas num plantio cercado por floresta.

Embora os botões florais tenham mostrado um desenvolvimento rápido, as regressões múltiplas entre o número de flores e as variáveis climáticas não identificaram claramente um intervalo de tempo no qual se possa afirmar 

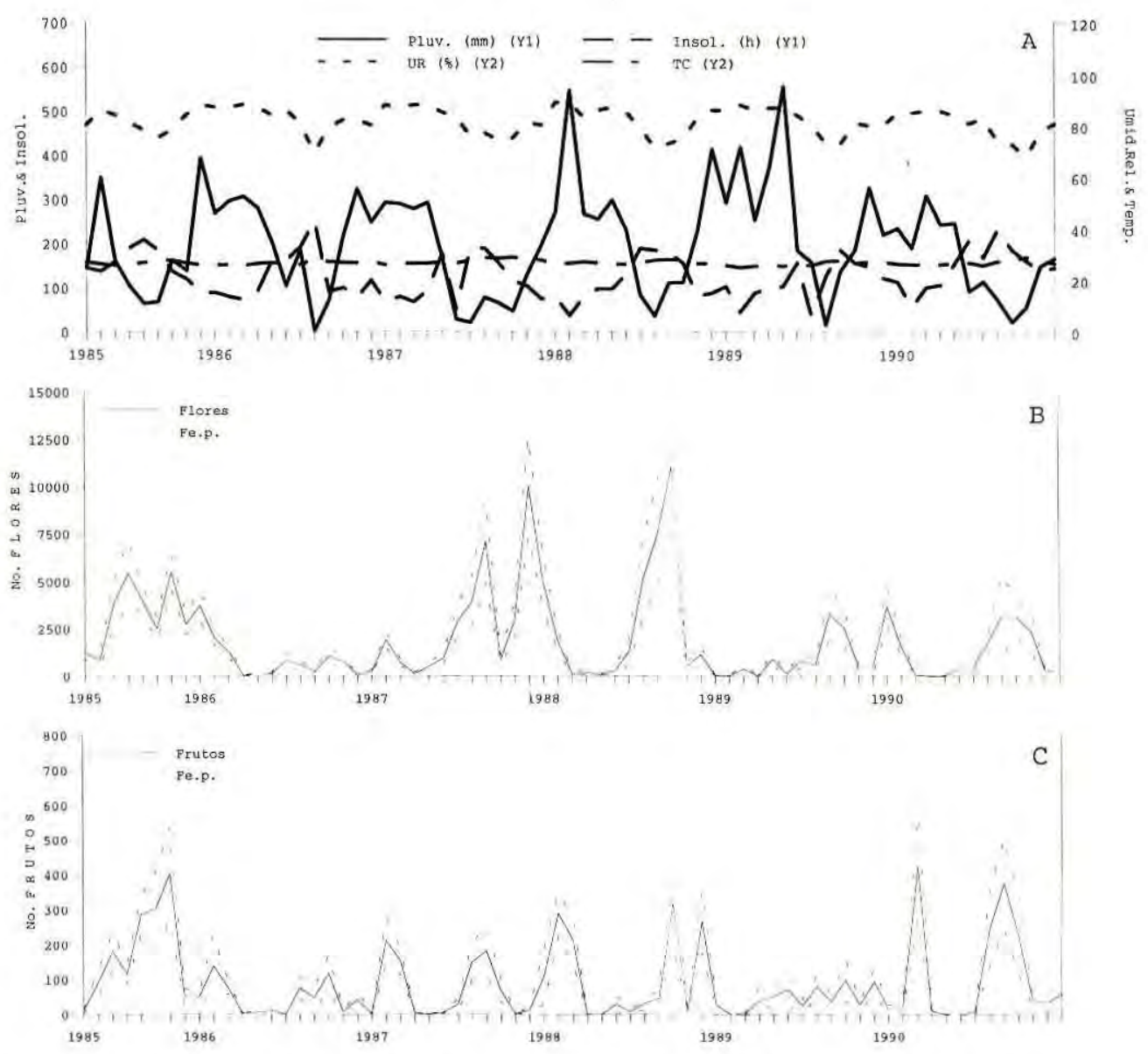

Figura 1. A. Dados climáticos de Manaus, Amazonas, Brasil, de abril de 1985 a dezembro de 1990. B. Número médio mensal ( \pm erro padrão) de flores de araçá-boi (Eugenia stipitata) no periodo. C. Número médio mensal (土e.p.) de frutos de araça-boi no periodo.

que houve o primeiro estimulo à floração (Tab. 1). A variação climática do mesmo mês apresentou o melhor modelo $\left(\mathrm{r}^{2}=0,20 ; \mathrm{s}=2100 ; \mathrm{p}=\right.$ $0,001)$, mas nenhuma das variáveis climáticas foi significativa (pelo testet) mesmo que a temperatura tenha respondido por $80 \%$ da variação no modelo. Isto sugere que o primeiro estimulo deveria ter ocorrido antes. Embora o modelo tenha sido significativo, o $\mathrm{r}^{2}$ é pequeno, provavelmente devido ao número e tamanho variável de picos de floração durante o ano ao longo do período de observações, e é similar aos $\mathrm{r}^{2} \mathrm{~s}$ observados por Alencar (1994) em diversas Sapotaceae na Reserva Florestal A. Ducke, Manaus, AM.

A variação climática do mês anterior (Flores-1; Tab. 1) apresentou um modelo um pouco menos significante 
Tabela 1. Regressões multiplas* entre o número de flores de araçá-boi (Eugenia stipitata) e as variáveis climáticas mensais de Manaus, AM, Brasil, de 1985 a 1990, feitas com 0, - 1, - 2 ou - 3 meses de diferência entre as variáveis climáticas e a floração para identificar o possivel periodo de iniciação floral.

\begin{tabular}{|c|c|c|c|c|c|c|c|}
\hline & Constante & Temp, ${ }^{\circ} \mathrm{C}$ & Pluv. (mm) & U.R. (\%) & Insol. (h) & s & $\mathrm{R}^{2}$ \\
\hline Flores0 ** & $-13.381^{\mathrm{ms}}$ & $+645 T^{n a}$ & $-4,89 P^{n s}$ & $-14 U^{\circ s}$ & $+1,15$ rs $^{\text {s. }}$ & 2100 & 0,20 \\
\hline$\%$ var. ${ }^{1}$ & & $80,6 \%$ & $19,1 \%$ & $0,2 \%$ & $0,1 \%$ & & \\
\hline Flores-1 = & $11.487^{\mathrm{ns}}$ & $-145 T^{10}$ & $-7,47 \mathrm{P}^{*}$ & $-54 \cup R^{n s}$ & $+1,75 \mathrm{~m}^{\mathrm{ma}}$ & 2130 & 0,18 \\
\hline$\%$ var. & & $41,2 \%$ & $56,6 \%$ & $1,9 \%$ & $0,3 \%$ & & \\
\hline Flores-2* $=$ & $-17.308^{63}$ & $+194 T^{\text {nis }}$ & $-7,46 \mathrm{P}^{\circ}$ & $+172 \mathrm{UR}^{\mathrm{ns}}$ & $+11,81^{1=}$ & 2243 & 0,09 \\
\hline$\%$ var. & & $10,3 \%$ & $58,1 \%$ & $8,8 \%$ & $22,8 \%$ & & \\
\hline Flores $-3^{* *}=$ & $-61.395^{* *}$ & $+1.168 \mathrm{~T}^{*}$ & $-2,44 P^{\text {ns }}$ & +363 UR $^{* *}$ & $+23.31^{*}$ & 2170 & 0,15 \\
\hline$\%$ var. & & $7,3 \%$ & $1,0 \%$ & $29,2 \%$ & $62,5 \%$ & & \\
\hline
\end{tabular}

I - \% da variaçào explicada por cada variável na regressão

* - significante a $p<5 \%$ pelo t-teste do modelo. ns - não significante a nivel de $p=5 \%$, ${ }^{*}$ - significante a $p<1 \%$ pelo t-teste do modelo

$\left(\mathrm{r}^{2}=0,18 ; \mathrm{s}=2130 ; \mathrm{p}=0,002\right)$, mas a pluviosidade foi uma variável significante $(p=0,034)$ e teve um peso negativo no modelo. Isto sugere que 0 primeiro estimulo ocorre entre 30 e 60 dias antes da abertura da flor, mais os modelos de dois e três meses antes da floração também apresentaram tendências que sugerem que o primeiro estimulo poderia ser mais cedo ainda. Dois meses antes da floração (Flores-2; Tab. 1) a variável pluviosidade foi significante no modelo $(\mathrm{p}=0,042)$, embora o modelo como um todo tenha apresentado uma menor significância. Três meses antes da floração (Flores-3; Tab. 1), as variáveis umidade relativa $(\mathrm{p}=0,003)$ e insolação $(p=0,003)$ foram altamente significantes e a variável temperatura $(\mathrm{p}$
$=0,028)$ foi significante, embora $o$ modelo tenha apresentado uma menor significância do que o modelo de Flores-1. O modelo de Flores-4 (não apresentado) não foi significante ( $\mathrm{p}>$ $0,45)$, sugerindo que o clima de quatro meses antes da floração não tem efeito na iniciação floral.

A variação na importância de cada variável climática e os pequenos coeficientes de determinação não permitem uma conclusão sobre que variável é o primeiro estimulo à floração, nem quando isto ocorre. Uma análise mais detalhada, comparando dados climáticos semanais com dados de floração semanais, talvez poderá identificar o primeiro estimulo.

As flores que foram isoladas em sacos de morim nunca vingaram 
frutos. Falcão et al. (1988) observaram uma taxa de auto-fecundação de $2 \%$, sugerindo que nas plantas juvenis estudadas por eles a auto-fecundação ocorreu como resultado da juvenilidade das plantas e não porque o araçá-boi naturalmente se autofecunda. A falta de auto-fecundação observada confirma que a estrutura floral de plantas adultas não permite auto-polinização não-assistida. Serão necessários futuros estudos para determinar o grau de auto-fecundação assistida.

\section{Frutificação}

A frutificação ocorreu logo após à floração (Fig. 1C). Embora o magnitude da floração tenha sido muito variável ao longo dos anos, a frutificação foi um pouco menos variável. Os picos maiores (setembro a novembro 1985 , janeiro a março 1988, março e agosto a outubro 1990) produziram entre 300 e 400 frutos por planta e ocorreram no final da estação seca (agosto a outubro), exceto em 1988 e 1990 quando houve picos similares em magnitude ocorrendo na estação chuvosa (janeiro a março). Nos anos de menores picos (1986, 1989), as plantas produziram entre 100 e 200 frutos e os picos foram distribuidos mais uniformemente ao longo do ano, especialmente em 1989. Portanto, tanto no período seco como no periodo chuvoso frutos estavam presentes, como também observaram Falcão et al. (1988) e Pinedo et al. (1981). Falcão et al. (1988) observaram que as plantas juvenis produziram entre 50 e 200 frutos nos picos maiores, que certamente é uma consequência da juvenilidade.

Embora não tenha sido coletadas informações sistemáticas sobre o tamanho dos frutos, observou-se que no periodo de maior precipitação os frutos foram maiores que no periodo seco. Esta variação sazonal de tamanho explica a observação de Kanten (1994) de que há maior produção na época chuvosa do que na época seca.

Falcão et al. (1988) observaram que o tempo entre a fecundação e a maturação dos frutos foi de aproximadamente 54 dias (publicado erradamente como 34 dias), embora Galvis \& Hernández (1993) tenham observado um intervalo de 72 dias. Neste trabalho observamos que este intervalo foi de aproximadamente 56 dias em 1988. Supõe-se que esta variação (de 54 a 72 dias) pode ser devida às condições nutricionais das plantas e à variação climática no periodo de observação, embora estudos mais detalhados serão necessários para confirmar isto.

As regressões múltiplas entre a frutificação e as variáveis climáticas sugerem que a maturação leva mais de 30 dias e menos de 60 , pois a regressão com as variáveis climáticas do mesmo mês (Frutos-0; Tab. 2) não foi significante, enquanto a regressão com as variáveis climáticas do mês anterior (Frutos-1) foi significante $\left(\mathrm{r}^{2}\right.$ $=0,14 ; p=0,009)$. Nesta regressão a pluviosidade explicou $73 \%$ da variação do modelo, embora seu efeito tenha sido negativo e não significativo 
pelo teste de $t(p=0,106)$. A regressão com dados de dois meses anteriores (Frutos-2) não foi significativa, nem a de três meses, que teve um $\mathrm{p}>0,50$ (não apresentado). Sugere-se que estudos mais aprofundados em diferentes épocas do ano com plantas sempre bem nutridas possam identificar este periodo com maior precisão.

\section{Mudança Foliar}

A queda das folhas ocorreu sempre perto do final da frutificação e o lançamento de folhas novas ocorreu antes do inicio da próxima floração, confirmando as observações de Falcão et al. (1988) em plantas juvenis. Isto segue o padrão de outras fruteiras amazônicas, tais como araçá-pera (Psidium acutangulum; Falcão et al., 1992), umari (Poraqueiba sericea; Falcão \& Lleras, 1980) e pajurá (Couepia bracteosa; Falcão et al., 1981).

\section{Vingamento de Frutos}

Falcão et al. (1988) verificaram que a porcentagem de vingamento de frutos foi de $25 \%$ em 1983 em plantas juvenis adubadas. No presente estudo, com plantas crescendo em solo degradado, o vingamento anual variou de 3,5 a $12,5 \%$ (Fig. 2). Nos primeiros anos (1985-87) o vingamento foi sempre menor e menos variável. No restante do periodo o vingamento foi maior e muito variável. Supõe-se que o menor vingamento foi devido às condições agronômicas menos adequadas no presente estudo e não à polinizaçâo, pois sempre haviam abelhas na parcela experimental.

$\mathrm{O}$ vingamento anual parece seguir a variação pluviométrica (Fig. 2). No entanto, a regressão múltipla usando as médias anuais não foi significativa (vingamento $=57+6,6 \mathrm{~T}$ $+0,134 \mathrm{P}-3,31 \mathrm{UR}+0,137 \mathrm{I} ; \mathrm{r}^{2}=0,15$;

Tabela 2. Regressões multiplas* entre o número de frutos de araçá-boi (Eugenia stipitata) e as variáveis climáticas mensais de Manaus, AM, Brasil, de 1985 a 1990, feitas com 0, - 1 ou - 2 meses de diferência entre as variáveis climáticas e a frutificação para identificar o possível periodo de maturação.

\begin{tabular}{|c|c|c|c|c|c|c|c|}
\hline & Constante & Temp. ${ }^{\circ} \mathrm{C}$ & Pluv. (mm) & U.R. (\%) & Insol. (h) & $\mathrm{s}$ & $\mathrm{R}^{2}$ \\
\hline Fruto0 $0^{\text {ns }}=$ & $1.160^{\mathrm{ns}}$ & $-21,4 T^{\text {ns. }}$ & $-004 \mathrm{P}^{\mathrm{n}}$ & $-6,0 \mathrm{UR}^{\mathrm{ns}}$ & $+0,031^{15}$ & 110,5 & 0,0 \\
\hline$\%$ var.1 & & $21,0 \%$ & $39,9 \%$ & $39,9 \%$ & $0,1 \%$ & & \\
\hline Frutos $-1^{* *}=$ & $1.234^{\text {ns }}$ & $-37,2 T^{\mathrm{ns}}$ & $-027 P^{n s}$ & $-2,03 \cup R^{n s}$ & $+0,55 \mathrm{p}^{\mathrm{ss}}$ & 102,2 & 0,14 \\
\hline$\%$ var. & & $3,3 \%$ & $72,9 \%$ & $6,8 \%$ & $17,0 \%$ & & \\
\hline Frutos-2 $2^{\text {ns }}=$ & $407^{\text {ns }}$ & $-18,6 T^{n s}$ & $-0,22 P^{n s}$ & $+1,74 \mathrm{UR}^{\mathrm{rs}}$ & $+0,65 h^{\mathrm{ss}}$ & 106,5 & 0,08 \\
\hline$\%$ var. & & $3,5 \%$ & $61.6 \%$ & $1.4 \%$ & $33,5 \%$ & & \\
\hline
\end{tabular}

1 - \% da variaçāo explicada por cada variável na regressăo

* - significante a $p<5 \%$ pelo t-teste do modelo, ns - năo significante a nivel de $p=5 \%$; ${ }^{* *}$ - significante a $p<1 \%$ pelo t-teste do modelo. 
$\mathrm{p}=0,58)$. A correlação entre vingamento e pluviosidade foi baixa $(\mathrm{r}=0,30)$, menor do que a correlação com umidade relativa do ar $(r=-0,47)$. Outra vez é provável que as correlações e coeficiente de determinação foram baixas devido à grande variação de floração e frutificação ao longo dos anos.

\section{Produtividade}

$\mathrm{O}$ peso médio dos frutos (135 g) variou muito de planta para planta em 1988 (Tab. 3), com um minimo de $95 \mathrm{~g}$ e um máximo de $180 \mathrm{~g}$. No plantio de araçá-boi que deu origem ao plantio estudado no presente trabalho, Ferreira (1992) observou um peso médio de 161 g em julho de 1985, com um mínimo de $50 \mathrm{~g}$ e um máximo de $585 \mathrm{~g}$. A diferença entre as observações é provavelmente devido as variações climáticas, manejo das plantas (especialmente adubação), e maior variabilidade do germoplasma original.

Os componentes físicos dos frutos (casca, semente, polpa) variaram bastante entre as plantas $(\mathrm{CV}$ de $20 \%$ para polpa a $30 \%$ para semente), mas a porcentagem de polpa $(78 \%)$ variou muito menos (CV de $5 \%$ ). A porcentagem de polpa encontrada é intermediária entre as porcentagens encontradas por Ferreira (1992) e Pinedo et al. (1981), de 63\% e $69 \%$, respectivamente, e Andrade $e t$ al. (1989), de $85 \%$. Esta variação na literatura é provavelmente devida a diferenças no germoplasma, no manejo e nas condições climáticas.

O número de sementes variou de 6 a 13, com média de 9 (Tab. 3), cada uma pesando ao redor de $2,4 \mathrm{~g}$. Ferreira (1992) encontrou uma média de 12 sementes, pesando em média 2,8 g, outra vez sugerindo a importância de um manejo adequado, embora este também requer mais estudo para confirmar.

A estimativa do número de frutos produzidos por planta por ano variou de um mínimo de 15 (árvore $10 \mathrm{em}$ 1989) a um máximo de 4.053 (árvore

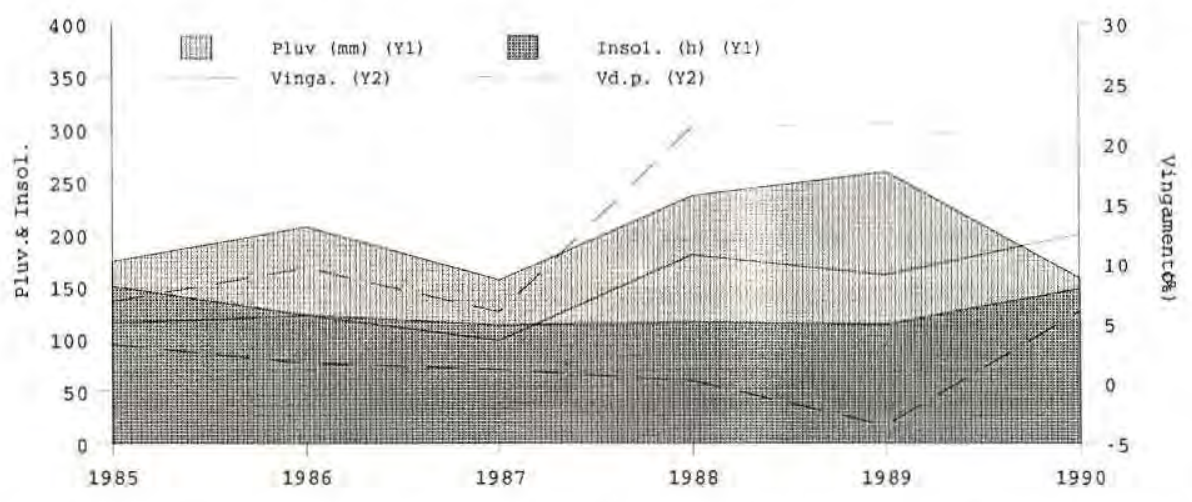

Figura 2. Vingamento médio anual ( \pm desvio padrão) de frutos de araçá-boi (Eugenia stipitata) de 1985 a 1990, com as médias anuais de pluviosidade mensal $(\mathrm{mm})$ e insolação mensal (h) no periodo. 
5 em 1990), com uma média \pm desvio padrão de $1.029 \pm 664$ frutos e uma mediana de 892 frutos; novamente vale ressaltar que o uso da regra de três poderia super ou sub-estimar estes números. Como aconteceu com outras variáveis, a variação do número de frutos foi grande entre as plantas e entre os anos (Fig. 3), com uma distribuição anormal. Metade das árvores sempre produziu pouco, enquanto três quase sempre produziram muito, sugerindo um efeito do solo ou do genótipo.

\section{Insetos visitantes}

Observou-se a presença diária de abelhas quando haviam flores no plantio. Essas chegavam as cinco horas da manhã concomitante com a abertura dos botões florais mas cada espécie visitava as flores em um horário diferente. Em dias chuvosos a frequência de abelhas foi menor e em dias ensolarados haviam um número maior delas.

As abelhas mais frequentes foram Apis mellifera, Eulaema mocsaryi, e Ptilotrigona lurida (Tab. 4), com as outras sendo menos frequentes. Em geral, as abelhas observadas por Falcão et al. (1988) em um ecossistema diferente foram as mesmas agora observadas na zona urbana.

As abelhas coletadas apresentaram bastante pólen de araçá-

Tabela 3. Variação de peso dos frutos e seus componentes dentre e entre plantas de araçá-boi (Eugenia stipitata) em Manaus em janeiro 1988.

\begin{tabular}{|c|c|c|c|c|c|c|c|}
\hline \multirow[b]{2}{*}{$\operatorname{Arrv}(n)$} & \multicolumn{4}{|c|}{ Peso $(\mathrm{g})$} & \multirow{2}{*}{$\begin{array}{c}\% \\
\text { Polpa }\end{array}$} & \multicolumn{2}{|c|}{ Sementes } \\
\hline & Fruto & Casca & Semente & Polpa & & Número & Peso \\
\hline $1(18)$ & $131,0 \pm 40,8$ & $9,6 \pm 2,4$ & $19,5 \pm 8,9$ & $102,0 \pm 32,1$ & $77,7 \pm 4,4$ & $8,8 \pm 3,7$ & $2,3 \pm 0,5$ \\
\hline $2(13)$ & $108,5 \pm 50,6$ & $5,2 \pm 1,7$ & $23,1 \pm 11,9$ & $80,2 \pm 38,0$ & $73,8 \pm 3,8$ & $12,8 \pm 5,2$ & $1,9 \pm 0,6$ \\
\hline $3(5)$ & $144,7 \pm 54,8$ & $8,6 \pm 3,0$ & $22,9 \pm 11,2$ & $113,2 \pm 46,6$ & $77,4 \pm 10,7$ & $12,2 \pm 4,4$ & $1,8 \pm 0,5$ \\
\hline $4(8)$ & $123,4 \pm 40,1$ & $7,7 \pm 3,6$ & $17,6 \pm 8,8$ & $98,2 \pm 38,8$ & $78,4 \pm 6,9$ & $6,5 \pm 2,2$ & $3,0 \pm 1,7$ \\
\hline $5(18)$ & $172,4 \pm 87,2$ & $12,0 \pm 6,2$ & $20,4 \pm 12,6$ & $140,0 \pm 75,0$ & $80,8 \pm 7,6$ & $8,9 \pm 4,8$ & $2,8 \pm 1,9$ \\
\hline $6(36)$ & $119,7 \pm 59,6$ & $8,6 \pm 3,6$ & $20,2 \pm 12,3$ & $90,8 \pm 46,0$ & $74,1 \pm 13,3$ & $9,9 \pm 4,1$ & $2,4 \pm 1,4$ \\
\hline $7(16)$ & $139,3 \pm 76,6$ & $9,4 \pm 3,1$ & $16,7 \pm 12,9$ & $113,2 \pm 63,1$ & $80,9 \pm 3,7$ & $6,2 \pm 3,3$ & $2,6 \pm 1,1$ \\
\hline $8(15)$ & $94,8 \pm 44,6$ & $6,6 \pm 2,2$ & $8,1 \pm 4,4$ & $80,2 \pm 40,0$ & $83,6 \pm 5,6$ & $3,9 \pm 2,1$ & $2,4 \pm 1,6$ \\
\hline $9(11)$ & $180,0 \pm 64,9$ & $9,8 \pm 3,0$ & $32,6 \pm 15,2$ & $137,6 \pm 51,4$ & $76,1 \pm 4,1$ & $13,4 \pm 2,9$ & $2,5 \pm 1,3$ \\
\hline $10(12)$ & $130,8 \pm 63,1$ & $9,8 \pm 3,1$ & $27,5 \pm 19,7$ & $93,5 \pm 42,0$ & $72,4 \pm 5,8$ & $9,8 \pm 2,1$ & $2,6 \pm 1,4$ \\
\hline Média & $134,5 \pm 26,4$ & $8,7 \pm 1,9$ & $20,9 \pm 6,5$ & $104,9 \pm 21,3$ & $77,5 \pm 3,6$ & $9,2 \pm 3,1$ & $2,4 \pm 0,4$ \\
\hline
\end{tabular}


boi em suas patas, confirmando sua atividade coletora nas flores. A ausência de outros polinizadores aparentes sugere que as abelhas sejam as principais polinizadoras do araçá-boi.

\section{CONCLUSÕES}

$\mathrm{O}$ araçá-boi floresce e frutifica durante a maior parte do ano, com vários picos de intensidades diferentes. A iniciação floral é um evento complexo e demorado que pode levar de dois a três meses, embora o periodo entre o aparecimento do botão floral e sua antese seja curto ( 15 dias). O período de floração até a maturação dos frutos dura entre 50 e 60 dias. As regressões múltiplas usadas para determinar o efeito das variáveis climáticas na floração e frutificação não tiveram altos coeficientes de determinação, embora os modelos tenham sido significantes, provavelmente porque o araçá-boi floresce numerosas vezes ao longo do ano e ainda não se sabe qual o

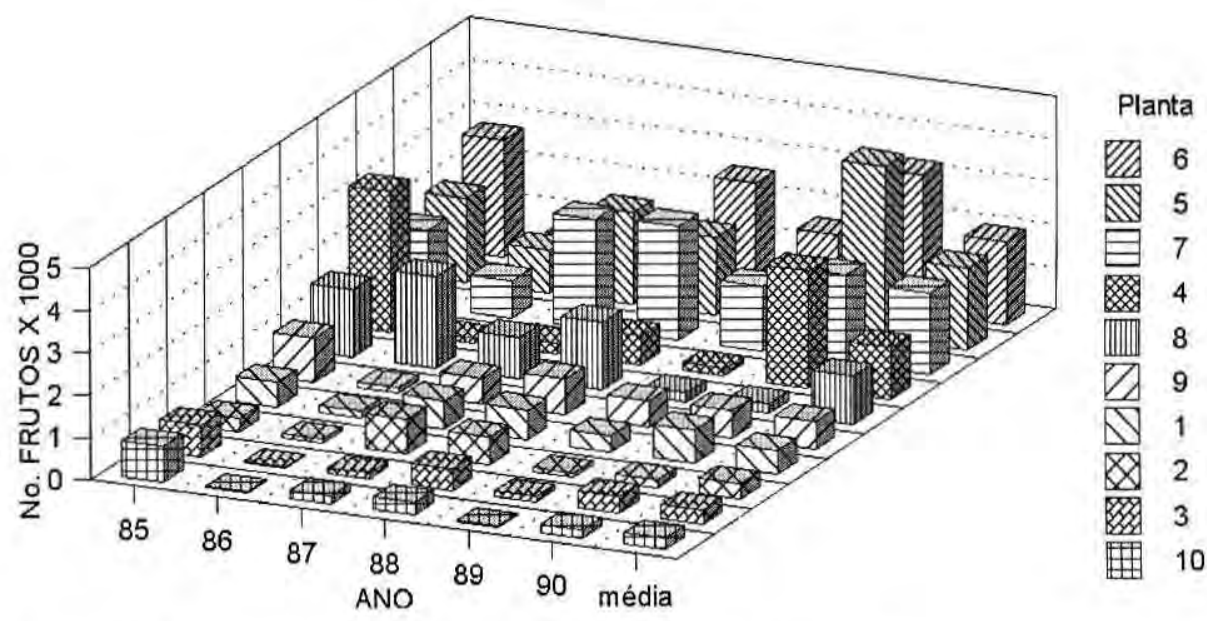

Figura 3, Variação no número estimado de frutos de araçá-boi (Eugenia stipitata) produzidos por planta por ano de 1985 a 1990 num plantio em latossolo amarelo degradado e manejado de forma irregular em Manaus, Amazonas. As plantas são ordenadas na figura conforme o número médio de frutos no periodo.

Tabela 4. Insetos visitantes às flores de araçá-boi (Eugenia stipitata) ao longo do ano de 1988 em Manaus, AM, Brasil.

\begin{tabular}{lll}
\hline Ordem & Familia & Espécie \\
\hline Hymenoptera & Apidae & Apis mellifera scutellata Lepeletier, 1836 \\
& & Eulaema bombiformis Packard, 1869 \\
& Eulaema mocsaryi Friese, 1899 \\
& Melipona fulva Lepeletier, 1836 \\
& Melipona seminigra merrillae Cockerell, 1919 \\
& Partamona sp. \\
Diptera & Ptilotrigona lurida mocsaryi Friese, 1900 \\
& Ornidia obesa \\
\hline
\end{tabular}


estímulo mais importante para iniciar o processo. O vingamento foi variável ao longo do tempo, de menos de $5 \%$ até próximo de $15 \%$, com um efeito pequeno do clima.

\section{AGRADECIMENTOS}

Pesquisa feita com apoio do convênio Universidade do Amazonas, Instituto Nacional de Pesquisas da Amazônia entre 1982 e 1990, e do Conselho Nacional de Desenvolvimento Científico e Tecnológico entre 1997 e 1999.A primeira autora agradece ao Conselho Nacional de Desenvolvimento Científico e Tecnológico (CNPq) e ao Instituto Nacional de Pesquisas da Amazônia (INPA) pela bolsa DTI concedida. Os autores agradecem ao Dr. Francisco J. Aguilera Peralta pela identificação dos insetos visitantes, ao técnico José Rufino pelo apoio no campo, a Dr ${ }^{\mathrm{a}}$ Marlene F. Silva e dois referees anônimos pela revisão critica do manuscrito.

\section{Bibliografia citada}

Alencar, J.C. 1994. Fenologia de cinco espécies arbóreas tropicais de Sapotaceae correlacionada a variáveis climáticas na Reserva Ducke, Manaus, AM. Acta Amazonica, 24(3/4): 161-182.

Andrade, J.S.; Aragão, C.G.; Chaar, J.S.; Leão, I.M.S. 1989. Caracterização do araçá-boi (Engenia stipitata sub-esp. sororia McVaugh). Anais do Congresso Brasileiro de Ciencia e Tecnologia de Alimentos, 12: 87.

Calzada B., J, 1980. 143 Frutales Nativas. Libreria El Estudiante, Lima, Peru. 320p.

Cavalcante, P.B. 1991. Frutas Comestiveis da Amazônia, $5^{a}$ Ed. Edições CEJUS/Museu Paraense Emilio Goeldi, Belém, Pará. 279 p.
Chávez F., W.B.; Clement, C.R. 1984. Considerações sobre o araçá-boi (Eugenia stipitata McVaugh, Myrtaceac) na Amazônia Brasileira. Anais do Congresso Brasileiro de Fruticultura, 7: 167-177.

Clement, C.R. 1989. A center of crop genetic diversity in western Amazonia. BioScience, 39(9): 624-631.

Erdtman, G. 1960. The acetolysis method, a revised description. Bot. Tidskn. Lund., 54(4): 561-664.

Falcão, M.A. (ed.). 1983. Aspectos fenológicos, ecológicos e de produtividade de algumas fruteiras cultivadas na Amazônia, Vol. I. Editora Umberto Calderaro e SUFRAMA, Manaus, Amazonas. 201p.

Falcão, M.A.; Lleras, E. 1980, Aspectos fenológcos, ecológicos e de produtividade do umai (Poraqueiba sericea Tul.). Acta Amazonica, 10(3): 445-462.

Falcão, M.A.; Lleras, E; Kerr, W.E. 1981. Aspectos fenológícos, ecológicos e de produtividade do pajurá (Couepia bracteosa Benth.). Acta Amazonica, 11(3): 473-482.

Falcão, M.A.; Chavéz F., W.B.; Ferreira, S.A.N.; Clement, C.R.; Barros, M.J.B.; Brito, J.M.C.; Santos, T.C.T. 1988. Aspectos fenológicos e ecológicos do araçá-boi (Eugenia stipitata McVaugh) na Amazônia Central. I. Plantas juvenís, Acta Amazonica, 18(3-4): 27-38.

Falcão, M.A.; Ferreira, S.A.N.; Clement, C.R.; Santos, T.C.T.; Medeiros, R.S. 1992. Desenvolvimento e fenologia de plantas de araçá-pera (Psidium acutangulum DC) na Amazônia central brasileira. Acta Amazonica, 22(3): 285-293.

Ferreira, S.A.N. 1992, Biometria de frutos de araçá-boi (Eugenia stipitata McVaugh). Acta Amazonica, 22(3): 295-302.

Galvis V., J.A.; Hernández, M.S. 1993. Analisis del crecimento del fruto $y$ determinación de cosecha del arazá (Eugenia stipitata). Colombia Amazonica, 6(2):107-121.

Gentil, D.F.O; Clement, C.R. 1997. The araza (Eugenia stipitata): results and research 
directions. In: Donadio, L.C. (ed.). Proceedings of the International Symposium on Myrtaceae. Acta Horticulturae, 452: 9-17.

Kanten, R.F. van. 1994. Produtividad y fenologia del araza (Eugenia stipitata McVaugh) bajo tres sistemas agroflorestales en Baja Talamanca, Costa Rica. Dissertação de Mestrado, Centro Agronómico Tropical de Investigación e Enseñanza (CATIE), Turrialba, Costa Rica. 50p.

McVaugh, R. 1956. Tropical American Myrtaceae. Fieldiana Botany, 29(3): 145228.

McVaugh, R. 1958. Eugenia stipitata. In: Flora of Peru. Field Museum Natural History - Botany, 13: 736-737.

Pinedo, P.M.; Ramirez, N.; Blasco, M.L. 1981. Notas preliminares sobre el araza (Eugenia stipitata), frutal nativo de la Amazonia Peruana. Pub. Misc. 229, Instituto Nacional de Investigación Agraria, Lima, Peru. 58p.

Ribeiro, M.N.G. 1976. Aspectos climáticos de Manaus. Acta Amazonica, 6(2): 229-233.

Sokal, R.R.; Rohlf, F.J. 1969. Biometry. W.H. Freeman, San Francisco. 690p.

Wielgolaski, F.E. 1974. Phenology in agriculture. In: Lieth, H. (ed.). Phenology and seasonality modeling. Chapman \& Hall, London. p. 369-381. 